\section{(2) OPEN ACCESS}

\title{
Tight control for Crohn's disease with adalimumab- based treatment is cost-effective: an economic assessment of the CALM trial
}

\author{
Remo Panaccione, ${ }_{1}^{1}$ Jean-Frederic Colombel, ${ }^{2}$ Simon P L Travis, ${ }^{3}$ Peter Bossuyt (i), ${ }^{4}$ \\ Filip Baert, ${ }^{5}$ Tomáš Vaňásek, ${ }_{1}^{6}$ Ahmet Danalıoğlu, ${ }^{7}$ Gottfried Novacek, ${ }_{1}^{8}$ \\ Alessandro Armuzzi, ${ }^{9}$ Walter Reinisch, ${ }^{10}$ Scott Johnson (1), ${ }^{11}$ Marric Buessing, ${ }^{12}$ \\ Ezequiel Neimark, ${ }^{13}$ Joel Petersson, ${ }^{13}$ Wan-Ju Lee, ${ }^{13}$ Geert R D'Haens ${ }^{14}$
}

- Additional material is published online only. To view please visit the journal online (http://dx.doi.org/10.1136/ gutjnl-2019-318256).

For numbered affiliations see end of article.

\section{Correspondence to} Dr Remo Panaccione, InflammatoryBowel Disease Unit, Division of Gastroenterology and Hepatology, Department ofMedicine, University of Calgary, 6D32 TRW Building 3280 Hospital Drive, Calgary, AB T2N 4N1, Canada ; rpanacci@ucalgary.ca

Received 10 January 2019 Revised 4 June 2019 Accepted 12 June 2019 Published Online First 8 July 2019
Check for updates

(C) Author(s) (or their employer(s)) 2020. Re-use permitted under CC BY-NC. No commercial re-use. See rights and permissions. Published by BMJ.

To cite: Panaccione $\mathrm{R}$, Colombel J-F, Travis SPL, et al. Gut 2020;69:658-664.

\begin{abstract}
Objective To evaluate the cost-effectiveness of an inflammatory biomarker and clinical symptom directed tight control strategy (TC) compared with symptombased clinical management (CM) in patients with Crohn's disease (CD) naïve to immunosuppressants and biologics using a UK public payer perspective.

Design A regression model estimated weekly CD Activity Index (CDAI)-based transition matrices (remission: CDAI $<150$, moderate: CDAI $\geq 150$ to $<300$, severe: $C D A l \geq 300$ to $<450$, very severe: $(D A I \geq 450$ ) based on the Effect of Tight Control Management on Crohn's Disease (CALM) trial. A regression predicted hospitalisations. Health utilities and costs were applied to health states. Work productivity was monetised and included in sensitivity analyses. Remission rate, CDrelated hospitalisations, adalimumab injections, other direct medical costs, quality-adjusted life-years (QALYS) and incremental cost-effectiveness ratio (ICER) were calculated.
\end{abstract}

Results Over 48 weeks, TC was associated with a higher clinical remission (CDAl $<150)$ rate $(58.2 \%$ vs $46.8 \%)$, fewer CD-related hospitalisations ( 0.124 vs 0.297 events per patient) and more injections of adalimumab (40 mg sc) (mean 31.0 vs 24.7) than CM. TC was associated with 0.032 higher QALYs and £593 higher total medical costs. The ICER was $£ 18656$ per QALY. The ICER was cost-effective in $57.9 \%$ of simulations. TC became dominant, meaning less costly but more effective, when work productivity was included. Conclusion A TC strategy as used in the CALM trial is cost-effective compared with CM. Incorporating costs related to work productivity increases the economic value of TC. Cross-national inferences from this analysis should be made with caution given differences in healthcare systems.

Trial registration number NCT01235689; Results.

\section{INTRODUCTION}

Crohn's disease (CD) is a chronic, disabling, progressive inflammatory disease of the gastrointestinal tract in which patients may develop strictures, abscesses or fistulas. ${ }^{1}$ Costs associated with $\mathrm{CD}$ are substantial. ${ }^{2} \mathrm{~A}$ review article estimated that the annual hospitalisation incidence due to $\mathrm{CD}$ was

\section{Significance of this study}

What is already known on this subject?

- Effect of Tight Control Management on Crohn's Disease was the first study to show that a biomarker-based and clinical symptom-based monitoring strategy (ie, tight control strategy (TC)) resulting in timely modification of adalimumab-based therapy prompts better endoscopic and clinical outcomes than relying on the conventional symptom-driven treatment strategy (CM) in patients with Crohn's disease (CD) naïve to immune suppressants and biologics.

What are the new findings?

- TC is cost-effective compared with CM. TC is associated with fewer hospitalisations and more time in remission. Total costs are higher in TC due to greater use of adalimumab and biomarker testing than CM but are offset by reduced hospitalisations and qualityadjusted life years gained, resulting in a cost-effectiveness ratio that indicates good value for money in the UK setting relative to published National Institute for Health and Care Excellence guidances.

How might it impact on clinical practice in the foreseeable future?

- Physicians should consider using an inflammatory biomarker-based and clinical symptom-based monitoring strategy to inform their treatment decisions for patients with moderate to severe active CD, given that TC is associated with superior efficacy compared with CM and is a cost-effective use of resources.

$20 \%$, half of patients with CD required surgery within 10 years after diagnosis and the risk of postoperative recurrence was $44 \%$ to $55 \%$ after 10 years. ${ }^{3}$ Severe CD has also been related to lower quality-of-life and work productivity, with indirect costs representing a substantial portion of the costs of CD. ${ }^{4}$ Direct costs were primarily driven by 
hospitalisation prior to the widespread use of biologics such as tumour necrosis factor inhibitors; biologic therapy is now the primary driver of direct costs. ${ }^{67}$ Despite the costs, biologic use is associated with significant improvement in efficacy and disease control compared with conventional therapy such as corticosteroids and immunomodulators. However, current treatment algorithms rely on symptoms alone to inform decisions which may delay initiation of the most effective treatment. ${ }^{8}$ These approaches may prolong use of corticosteroids and put patients at risk of infections, morbidity and mortality. ${ }^{9} 10$ A recent consensus stated that inflammatory biomarkers, such as faecal calprotectin (FC) and C-reactive protein (CRP), might be useful in detecting intestinal inflammation and could inform treatment adjustment. ${ }^{11}$

The Effect of Tight Control Management on Crohn's Disease (CALM) trial was a randomised, controlled, open-label, phase 3 study in 22 countries that assessed whether a tight control treatment strategy (TC) using inflammation biomarkers FC and CRP in addition to clinical symptoms to direct therapy was superior to conventional practice that did not rely on biomarkers to direct therapy, namely clinical management $(\mathrm{CM})$ in early $\mathrm{CD} .{ }^{12}$ The CALM trial evaluated adult patients with active endoscopic CD, a CD Activity Index (CDAI) of 150-450 depending on dose of prednisone at baseline and no previous use of immunomodulators or biologics. Detailed study design was published elsewhere. $^{12}$

CALM was the first study to show that a TC monitoring strategy and timely treatment modification with adalimumabbased therapy results in better clinical and endoscopic outcomes than conventional symptom-driven treatment strategy in patients with early CD. TC was associated with significant improvements in mucosal healing (defined as Crohn's disease endoscopic index of severity (CDEIS) $<4$ and no deep ulcers), deep remission and steroid-free remission in TC than CM at week $48 .{ }^{12}$

Costs associated with TC may include more frequent biomarker tests and biologic use. However, the clinical benefit of mucosal healing and deep remission associated with TC could also affect economic outcomes such as hospitalisation and work productivity gain, which can reduce healthcare and societal costs. This study aimed to analyse the hospitalisation and work productivity outcomes in CALM and examine the cost-effectiveness (CE) of TC and CM treatment strategies in an economic model using data from the CALM trial.

\section{METHODS}

\section{Model structure}

We developed a state transition (or Markov) model to compare TC and CM strategies with a UK public payer perspective using patient-observation data from the CALM trial, including the TC $(n=122)$ and CM $(n=122)$ groups. ${ }^{12}$ State transition models represent disease severity by health states defined by clinicallyrelevant severity measures. Patients in the model can transition from one health state to another based on transition probabilities. We based our health states on CDAI scores, which included remission (CDAI $<150$ ), moderate (CDAI $\geq 150$ to $<300$ ), severe $(\mathrm{CDAI} \geq 300$ to $<450)$ and very severe $(\mathrm{CDAI} \geq 450)$ with hospitalisation as a toll state (online supplementary figure S1). A sensitivity analysis was assessed including a mild state (CDAI $\geq 150$ to 220 ) (online supplementary appendix 1 ). Both 48 -week and 5-year time horizons were modelled. Models using these health states are based solely on disease activity, exclude opaque aspects of treatment response (eg, 'drug responsive') $)^{13}$ and have been published previously. ${ }^{14} 15$

\section{Data}

CALM trial data were analysed to derive model inputs for baseline health states, transition probabilities between health states, hospitalisation rates conditional on health states, adalimumab injections and indirect costs in terms of absenteeism (missed hours of work) due to CD. Wilcoxon rank-sum tests and $\chi^{2}$ tests compared continuous and categorical variables, respectively, in the TC and CM arms at baseline. Initial health state distribution was based on the pooled samples' CDAI scores.

CDAI was measured at weeks $0,2,6,11,23,35$ and 48 in the CALM trial. Patients were mapped into health states based on their CDAI at each observation. The model used an intentto-treat (ITT) approach. Missing CDAI values or censored values were imputed using the last-observation-carried-forward approach.

\section{Transition probabilities}

A regression model, specifically an ordered probit model, estimated weekly transition probabilities among health states. An ordered probit model allows for an outcome with multiple ordered categories (eg, remission, moderate, severe and very severe). A simple regression was estimated with a treatment variable (TC or $\mathrm{CM}$ ) to assess the treatment effect. To allow treatment effects to vary across health states and over time, the model used a specification where the treatment variable interacted with lagged health states. The model included covariates for time since last CDAI measure and a pre/post-week-23 dummy variable that corresponded to the peak clinical remission rates observed in CALM to improve extrapolation of outcomes outside the 48-week trial. All regressions employed robust standard errors clustered at the patient level. Predicted health state distributions were linearly interpolated between trial observations to derive weekly health state distributions. In sensitivity analysis, observed CDAI-based health state distributions were used instead of predicted distributions. Proportion of time in remission was calculated as the number of weeks in remission divided by total modelled weeks.

\section{Direct medical costs}

Direct medical costs comprised hospitalisation, other direct medical costs (including laboratory, radiology, emergency department, non-biologic drugs, physician consults and outpatient visits), adalimumab injections and biomarker tests using UK prices. The crude rate of CD-related hospitalisations observed in the CALM trial was reported in events per patient year for TC and CM. Cumulative hospital incidence and a simple probit were also estimated to assess the treatment effect. In the CE model, inputs of CD-related hospitalisation events were estimated from a multivariate probit model (using similar specifications as the ordered probit model for health states). CD-related hospitalisation costs were derived based on inpatient costs that stratified patients by disease severity. ${ }^{7}$

Estimates for other direct medical costs were derived from published research that stratified patients by disease severity. ${ }^{16}$ Costs of adalimumab were estimated using observed injections from the CALM trial and NHS prices from $2017 .{ }^{17}$ Patients in the TC group received CRP and FC tests at weeks 0,11 and every 12 weeks thereafter; costs included test costs, staff time and reader costs. ${ }^{18} 19$ All cost inputs were inflation adjusted to 2016/17 prices using the hospital community health services index. ${ }^{20}$ 


\section{Indirect costs/costs associated with absenteeism}

The Work Productivity and Activity Impairment (WPAI) questionnaire assessed subjects' ability to work and perform daily activities in terms of absenteeism due to $\mathrm{CD}$ at weeks 0,11 , 23, 35 and $48 .^{21}$ The effect of TC relative to CM was determined using an ordinary least squares model of the change in the percentage of missed hours out of a work week relative to baseline. Absenteeism was monetised using an average 2017 UK wage rate from the Office of National Statistics. ${ }^{22}$ Absenteeism was included in sensitivity analysis reflecting the average effect on costs for the total CALM sample.

\section{Health utility}

Quality-of-life was measured in the trial with the 36-Item Short Form Health Survey instrument at the same time points as the WPAI. ${ }^{23}$ Scores were transformed to Short-Form Six-Dimension (SF-6D) utilities. ${ }^{24}$ SF-6D scores that are transformed have been shown to have a smaller range and lower variance in values and floor effects. ${ }^{25}$ We used EQ-5D-based health utility from a larger study relating CDAI to estimate health state utility for the base case. ${ }^{26}$ We used the trial-based SF-6D-based estimates in a sensitivity analysis.

\section{Presentation of final results}

Cost-utility outcomes were calculated, including incremental CE ratio (ICER), incremental net monetary benefit (INMB), qualityadjusted life-years (QALYs) monetised at $£ 30000$ each and CE acceptability curves (CEAC). We conducted one-way sensitivity analyses on key variables to examine how results varied over plausible input ranges which were identified in the literature or via analysis of the CALM trial. This analysis re-runs our base-case model while individually varying the selected variables within defined input ranges, to provide an idea of how much certain parameters influence the ICER (table 1). The base-case analysis was also estimated outside the trial horizon to 2 and 5 years using modelling prediction based on 48 -week data from the CALM trial. To account for the uncertainty in all model parameters simultaneously, we conducted a probabilistic sensitivity analysis using 1000 second-order Monte Carlo simulations, which re-runs our primary analysis while varying parameter values simultaneously based on their distributions (table 1). We used the Cholesky decomposition of the covariance matrices from the multivariate regressions to account for correlated inputs in the probabilistic sensitivity analysis. ${ }^{27}$

\section{RESULTS}

The distribution of TC and CM arms across CDAI-based health states was not statistically significantly different at baseline (online supplementary table S1). The pooled distribution across CDAI-based health states at baseline was $74.2 \%$ moderate, $25.4 \%$ severe and $0.4 \%$ very severe. The ordered probit regression (online supplementary table S2 for simple specification and online supplementary table $\mathrm{S} 3$ for model specification) indicated that TC had a greater likelihood of patients maintaining or moving to a less severe health state; the Markov matrices estimated from these regressions and used in the model appear in online supplementary table S4.

TC-treated patients had 14 CD-related hospitalisations over 106.3 patient years (13.2 per 100 patient years) at week 48; CM-treated patients had $29 \mathrm{CD}$-related hospitalisations over 103.6 patient years $(28.0$ per 100 patient years, $\mathrm{p}=0.021)$. The hospitalisation rate from the regression analysis of CALM data indicated that more severe health states were associated with greater likelihoods of hospitalisation, and that TC was associated with a lower likelihood of hospitalisation (beta: -0.247 , $\mathrm{p}=0.037$, online supplementary table $\mathrm{S} 2$ ). Cumulative incidence

Table 1 Model inputs and values used in SA

\begin{tabular}{|c|c|c|c|c|c|c|c|c|}
\hline & Mean & SE & Alpha & Beta & Distribution & $\begin{array}{l}\text { High value in one- } \\
\text { way SA }\end{array}$ & $\begin{array}{l}\text { Low value in one- } \\
\text { way SA }\end{array}$ & Source \\
\hline CD-related hospitalisation costs, per admission & $£ 8573$ & 3429 & 6 & 1372 & Gamma & f10288 & f6859 & 7 \\
\hline \multicolumn{9}{|l|}{ Other direct medical costs, weekly* } \\
\hline Remission (CDAl <150) & f15 & 4 & 12 & 1 & Gamma & f23 & f0 & 16 \\
\hline Moderate (CDAI $\geq 150$ to $<300$ ) & $\mathrm{f} 42$ & 8 & & & Gamma & $\mathrm{f} 58$ & f0 & 16 \\
\hline Severe $(C D A I \geq 300$ to $<450)$ & $\mathrm{f} 66$ & 13 & & & Gamma & f91 & $\mathrm{f} 40$ & 16 \\
\hline CRP test & f1.97 & None & None & None & None & f5.53 & f1.97 & 1819 \\
\hline FC test & $£ 23.27$ & None & None & None & None & f67.93 & f23.27 & 18 \\
\hline \multicolumn{9}{|l|}{ Health utility, annual } \\
\hline Remission (CDAI <150) & 0.827 & 0.008 & 1747 & 366 & Beta & 0.843 & 0.810 & 26 \\
\hline Moderate (CDAI $\geq 150$ to <300) & 0.647 & 0.008 & & & Beta & 0.663 & 0.630 & 26 \\
\hline Moderate (CDAl $\geq 150$ to $<300$ ) & 0.742 & & 181 & 63 & Dirichlet & 1 & 0 & CALM $^{12} \dagger$ \\
\hline Severe $(C D A I \geq 300$ to $<450)$ & 0.254 & & 62 & 182 & Dirichlet & 0 & 0.5 & CALM $^{12} \dagger$ \\
\hline Very severe $(C D A I \geq 450)$ & 0.004 & & 1 & 243 & Dirichlet & 0 & 0.5 & CALM $^{12} \dagger$ \\
\hline
\end{tabular}

Transition probability, hospitalisation and absenteeism point estimates were for base-case analysis, parameters for one-way and probabilistic sensitivity analyses based on regression in online supplementary table $\mathrm{S1}$.

${ }^{*}$ National Health Service paid costs except hospitalisation and adalimumab.

tCALM trial data analysis.

CALM, Effect of Tight Control Management on Crohn's Disease trial; CD, Crohn's disease; CDAl, Crohn's Disease Activity Index; CRP, C-reactive protein; FC, faecal calprotectin; SA, sensitivity analysis. 


\section{A Observed cumulative incidence}

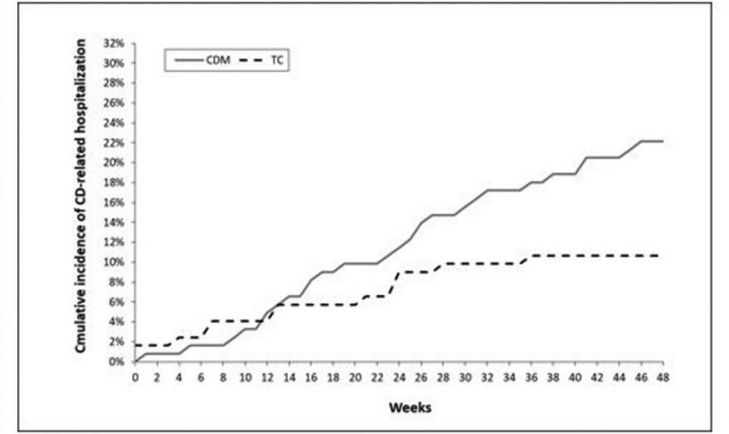

\section{B Modeled (ITT) cumulative incidence}

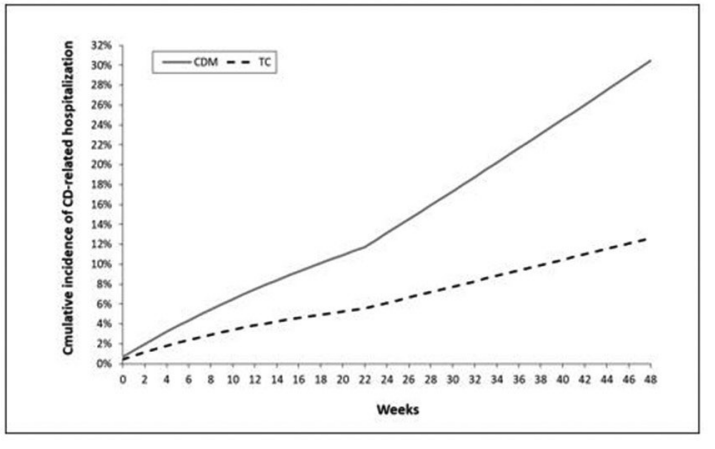

Figure 1 Observed and predicted cumulative incidence of CD-related hospitalisations. Cumulative incidence of CD-related hospitalisation based on observed cumulative hospitalisations in CALM (A) and modelled cumulative hospitalisations (B) based on regression in online supplementary table S3. CALM, Effect of Tight Control Management on Crohn's Disease trial; CD, Crohn's disease; ITT, intent-to-treat.

of hospitalisation appears in figure 1 ; in the ITT model, TC patients were predicted to have $0.124 \mathrm{CD}$-related hospitalisations compared with 0.297 for CM over 48 weeks.

The absenteeism regression, estimated with a sub-sample $(n=129)$ who recorded WPAI responses, revealed that TC was associated with a greater reduction of $5.6 \%$ in missed hours compared with baseline relative to $\mathrm{CM}$ over 48 weeks, although results were not statistically significant (beta: $-5.606, \mathrm{p}=0.346$ in simple model, online supplementary table S2).

The summary CE results over 48 weeks appear in table 2 . The model predicted that $58.2 \%$ of patients' 48 weeks were in clinical remission under the TC strategy, compared with $46.8 \%$ in the CM arm. TC-treated patients had 0.124 predicted hospitalisations per patient, CM had 0.297 , resulting in $£ 1462$ lower hospitalisation costs for TC-treated patients versus CM patients. Other direct medical costs were $£ 224$ lower for TC than CM patients. Mean adalimumab injections were 31.01 in TC and 24.74 in CM, which increased TC costs by $£ 2170$. The cost of CRP and FC testing increased TC costs by $£ 109$. QALYs were higher by 0.032 for TC patients. The ICER was $£ 18656$ per QALY, or $£ 360$ INMB, in the base case which excluded absenteeism.
TC reduced absenteeism by $£ 3962$, versus $-£ 2748$ for CM, which equates to about 77.5 more hours of work over 48 weeks per patient for TC. When absenteeism was included in the analysis, TC was the dominant treatment strategy (meaning less costly and more effective than CM), with $£ 1575$ INMB.

The results of the one-way sensitivity analysis appear in figure 2 . Model results were most sensitive to the baseline distribution of patients among $\mathrm{CD}$ severity levels, the inclusion of absenteeism, the costs of adalimumab and the health utility inputs. If all patients were in the moderate state at model baseline, the ICER was $£ 29695$ per QALY, indicating that TC was still preferred to CM but only marginally. If half of patients were severe and half very severe at baseline, TC was more effective and less costly (ie, dominant) versus $\mathrm{CM}$. The incremental costs of TC versus CM became negative (ie, less costly for TC) when including the costs related to absenteeism or when costs of adalimumab were reduced by $\geq 30 \%$, at which point TC become dominant. Using SF-6D converted health utilities resulted in an ICER of $£ 50910$ per QALY, suggesting that, counter to the base case, the CM strategy was more cost-effective than a TC strategy. If absenteeism was included and SF-6D utilities used, TC was dominant (£306 INMB). Modelling the base case beyond the end of the 48-week horizon by use of the Markov matrices

\begin{tabular}{|c|c|c|c|}
\hline Outcome & TC & $\mathrm{CM}$ & Incremental (TC-CM) \\
\hline Proportion of time in remission & 0.582 & 0.468 & 0.114 \\
\hline Adalimumab injections, $40 \mathrm{mg}$ & 31.01 & 24.74 & 6.27 \\
\hline \multicolumn{4}{|l|}{ Direct medical costs } \\
\hline Adalimumab costs & f10770 & f8601 & $£ 2170$ \\
\hline Other direct medical costs & f1332 & f1556 & $-\mathrm{f} 224$ \\
\hline Total costs & f13255 & f12662 & $\mathrm{f} 593$ (95\% Cl: $f-12952$ to $\mathrm{f} 2096)$ \\
\hline Total QALYs & 0.668 & 0.636 & $0.032(95 \% \mathrm{Cl}: 0.011$ to 0.055$)$ \\
\hline ICER (excluding absenteeism) & & & f18656 \\
\hline Incremental net monetary benefit (excluding absenteeism) & & & $£ 360$ \\
\hline
\end{tabular}

CM, Clinical management strategy; CRP, C-reactive protein; FC, faecal calprotectin; ICER, incremental cost-effectiveness ratio.; QALY, quality-adjusted life-year; TC, tight control strategy. 


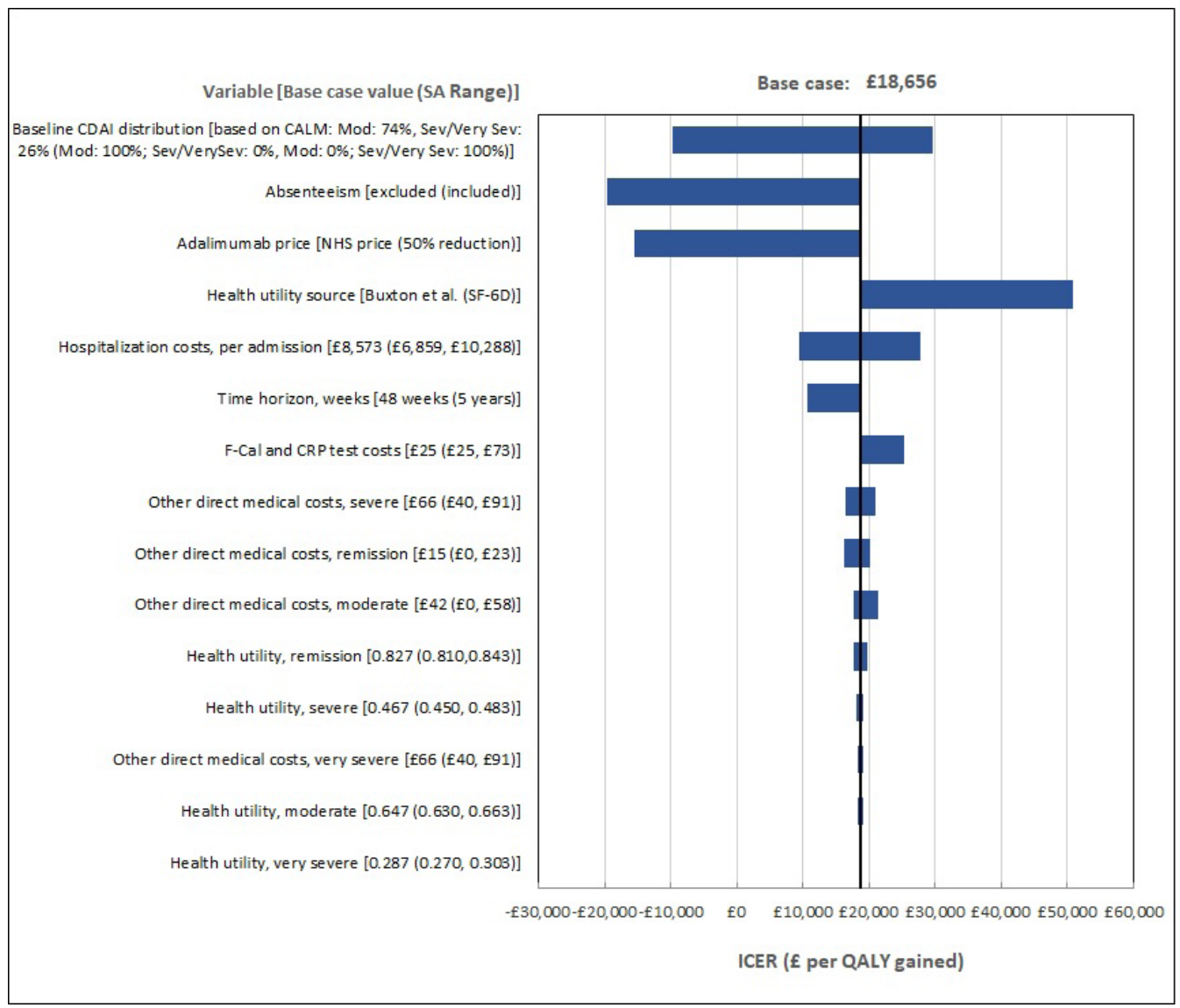

Figure 2 One-way sensitivity analysis of tight control versus clinical management base-case analysis. Values in base case and sensitivity analyses appear in table 1. CALM, Effect of Tight Control Management on Crohn's Disease trial CDAI, Crohn's disease Activity Index; ICER , incremental costeffectiveness ratio; NHS, National Health Service; SA, sensitivity analysis.

estimated by the regression model indicated that TC was more cost-effective at year 2 ( $£ 12166$ per QALY) and year 5 ( $£ 10675$ per QALY). The sensitivity analysis including a mild state resulted in a $£ 20991$ per QALY ICER (online supplementary appendix). The results of the PSA are displayed as a CEAC in figure 3. The CEAC indicated that $57.9 \%$ of simulations were below an ICER of $£ 30000$ per QALY in the base case (excluding absenteeism); $81.8 \%$ were below the same threshold when including absenteeism.

\section{DISCUSSION}

Although biologic use is a large component of CD treatment costs, our study showed that use of adalimumab with a TC management strategy brings significant clinical benefit in a costeffective way on a clinical and societal level. Using a model-based approach adopting data from the CALM trial, our study suggests that a TC strategy is cost-effective at traditional thresholds used by National Institute for Health and Care Excellence (ICERs below $£ 20000$ to $£ 30000$ per QALY). ${ }^{28}$ We found that the costs associated with greater use of adalimumab and additional CRP and FC testing in the TC strategy were offset by improvement in remission, reductions in CD-related hospitalisation and improvements in quality of life, which included resumed work productivity. Results were generally robust to sensitivity analyses, indicating that model estimates were reliable.

CALM incorporated economic endpoints and appropriate follow-up time for assessment of economic effects. It was not an event-based study or otherwise set up to hinder an ITT analysis. ${ }^{29}$ We maximised the patient-observation level data in the base case, using both modelled health states based on CDAI observations and unmodelled CDAI states in sensitivity analysis. Several resources not measured in the trial, including outpatient visits, emergency department, physician consults, radiology, comprehensive laboratory and non-biologic drugs were sourced from a study that stratified resource use by disease severity. ${ }^{16}$ The base-case analysis reflected the complete trial study period; sensitivity analysis projected observed trends outcomes onto longer time horizons.

The TC-based treatment strategy is cost-effective even though it involved greater adalimumab and testing use that lead to higher direct medical costs than conventional CM. In threshold analyses, the TC-based strategy would become not cost-effective if the adalimumab price increased by $16.6 \%$ to $£ 410.64$ per injection or if the FC test price were $358 \%$ above the base-case input ( $£ 106.77$ per test) in the UK setting. However, adalimumab cost is expected to decrease in anticipation of biosimilar therapy, which would increase the likelihood of CE of TC versus $\mathrm{CM}$, as shown in sensitivity analysis. Another key component of cost is hospitalisation episodes due to CD. In the trial, TC had a greater than $50 \%$ reduction in CD-related hospitalisation, offsetting some of the increased direct medical costs. When the time horizon of the model was adjusted to two and 5 years, the ICER decreased. Thus, the additional biologic and testing costs could be offset by further hospitalisation decrease at the margin.

The cost of absenteeism due to CD was the sole indirect cost included in the model. The WPAI included several other potentially monetisable endpoints, including impairment while working, overall work impairment and activity impairment. Across each WPAI measure, TC showed greater reductions in impairment than $\mathrm{CM}$ in every within-group (comparing study week versus baseline 


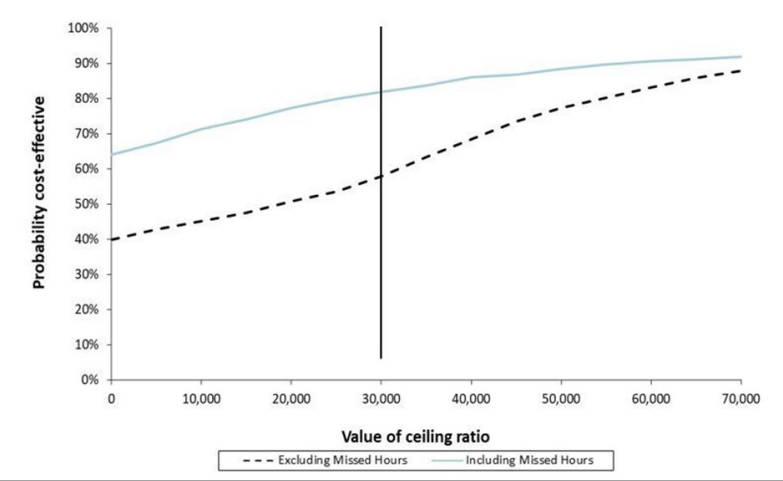

Figure 3 Cost-effectiveness acceptability curves (CEAC) of tight control versus clinical management excluding (base case) and including absenteeism due to Crohn's disease (CD) effects. To illustrate the uncertainty surrounding incremental cost-effectiveness ratio (ICER) estimates, the CEACs depict the probability that the tight control strategy (TC) strategy is preferred to the clinical management (CM) strategy across a range of cost-effectiveness ratios. Results are depicted for the analysis excluding absenteeism (hashed lines) and including absenteeism (solid grey line). Results are based on the probabilistic sensitivity analysis, which included 1000 second-order Monte Caro simulations in which model variables were simultaneously varied. The solid black vertical line indicates the $\mathrm{f} 30000$ per quality-adjusted life-years (QALY) willingness-to-pay threshold commonly used as a benchmark in the UK. $57.9 \%$ of simulations were at the threshold when excluding absenteeism, indicating that TC was likely better value considering costs and QALYs than CM; $81.8 \%$ were below the threshold when including absenteeism, indicating TC was more probably good value versus $\mathrm{CM}$ in that scenario.

WPAI outcomes) and between-group comparison at week 11, 23, 35 and 48; however, the between-group changes were not significant at an alpha of 0.05 with one exception. CALM may not have been powered to assess WPAI differences as only about half the participants were working at baseline. When including lost earnings from absenteeism due to $\mathrm{CD}$ using UK wage rates, the effects were large relative to direct medical costs. TC was dominant when these costs were included. These results are likely conservative as they excluded other measures of indirect cost.

If a biomarker-based and clinical symptom-based treatment strategy were superior to conventional CM in terms of efficacy, safety, quality of life and cost, will it replace CM as the new treatment paradigm? The results from this research could facilitate adoption of the TC practice style in the UK and perhaps other countries. There remain barriers to the adoption of new treatment strategies even when supported with trial-based evidence. Reimbursement for the FC test varies across primary care trusts in the UK. Diffusion of the TC practice pattern could be challenging in localities where the FC test is not reimbursed. Additionally, a new treatment strategy requires surmounting physician inertia to continue conventional practice patterns. Not modifying practice styles out of habit despite sound clinical and economic evidence to the contrary has been shown to be a major factor slowing uptake of new practice patterns. ${ }^{3031}$

There are limitations to this analysis. The CALM study population had early CD (median 2.61 months disease duration for both arms) which may not be generalisable to patients who have longer treatment histories. As a randomised controlled trial, observations from CALM came from an experimental setting, and may not be representative of typical patients with CD. This study used prices and a CE approach appropriate for the
UK which may not be transferable to other countries. Extrapolated results beyond the trial horizon per the sensitivity analysis cannot be externally validated. This analysis also focuses on average effects in the study population. Future research could evaluate whether some sub-groups may benefit more from TC.

\section{CONCLUSION}

Using CALM trial data, this study was the first to assess and demonstrate that use of adalimumab in a TC approach with both biomarker-based and clinical symptom-directed strategy is costeffective compared with CM from the UK perspective.

\section{Author affiliations}

${ }^{1}$ Inflammatory Bowel Disease Unit, University of Calgary, Calgary, Alberta, Canada

${ }^{2}$ Division of Gastroenterology, Icahn School of Medicine Vanasek, Division of

Gastroenterology and Hepatology, New York, New York, USA

${ }^{3}$ Gastroenterology, John Radcliffe Hospital, Oxford, UK

${ }^{4}$ Gastroenterology, Imelda General Hospital, Bonheiden, Belgium

${ }^{5}$ Department of Gastroenterology, University Hospitals Leuven, Leuven, Belgium

${ }^{6}$ Division of Gastroenterology and Hepatology, Hepato-Gastroenterologie HK, sro, Hradec Králové, Czech Republic

${ }^{7}$ Gastroenterology Department, Bezmialem Vakıf University, Istanbul, Turkey ${ }^{8}$ Department of Internal Medicine IV, Medical University of Vienna, Vienna, Austria

${ }^{9}$ Internal Medicine and Gastroenterology, Catholic University, Rome, Italy

${ }^{10}$ Department of Medicine IV, Medical University Vienna, Vienna, Austria

${ }^{11}$ Medicus Economics LCC., Milton, Maine, USA

${ }^{12}$ Medicus Economics, Boston, Massachusetts, USA

${ }^{13}$ AbbVie Inc., North Chicago, Illinois, USA

${ }^{14}$ Gastroenterology, AMC, Amsterdam, The Netherlands

Acknowledgements Medical writing support was provided by Suchin Virabhak and Julia Bond, Medicus Economics LLC, Boston, Massachusetts, USA

Contributors RP, J-FC, SPLT, PB, FB, TV, AD, GN, AA, WR, EN, JP, GD'H, W-JL: Study concept and design; analysis and interpretation of data; drafting of the manuscript; critical revision of the manuscript for important intellectual content; study supervision. SJ, MB: Study concept and design; acquisition of data; analysis and interpretation of data; drafting of the manuscript; critical revision of the manuscript for important intellectual content; statistical analysis; administrative, technical, or material support; study supervision. RP, J-FC, SPLT, PB, FB, TV, AD, GN, AA, WR, EN, JP, GD'H, W-JL, SJ, MB: approved the final draft submitted.

Funding Financial support for the study was provided by AbbVie. AbbVie participated in the interpretation of data, review and approval of the publication. All authors contributed to the development of the publication and maintained control over the final content.

Competing interests RP has received consultant and/or lecture fees from AbbVie, Amgen, AstraZeneca, Axcan Pharma (now Aptalis), Biogen Idec, Bristol-Myers Squibb, Centocor, ChemoCentryx, Eisai Medical Research Inc, Elan Pharmaceuticals, Ferring, Genentech, GlaxoSmithKline, Janssen, Merck Sharp \& Dohme Corp, Millennium Pharmaceuticals Inc (now Takeda), Ocera Therapeutics Inc, Otsuka America Pharmaceutical, Pfizer, Shire Pharmaceuticals, Prometheus Laboratories, Schering-Plough Corporation, Synta Pharmaceuticals Corp, Teva, UCB Pharma and Warner Chilcott. J-FC has been a consultant or advisory board member for AbbVie, Amgen, Boehringer-Ingelheim, Celgene Corporation, Celltrion, Enterome, Ferring, Genentech, Janssen and Janssen, Medimmune, Merck \& Co, Pfizer, Protagonist, Second Genome, Seres, Shire, Takeda, Theradiag, speaker for AbbVie, Ferring, speaker's bureau for Amgen, stock options of Intestinal Biotech Development, Genefit. Research Grants: AbbVie, Takeda, Janssen and Janssen. SPLT has been a consultant or advisory board member for AbbVie, Ajinomoto, Amgen, Almirall, Asahi, Atlantic, Bioclinica, Biogen, Boehringer Ingelheim, Bristol-Myers Squibb, Calcico, Celgene, Chemocentryx, Cosmo, Covance, Enterome, Falk, Ferring, Genentech, Gilead, Giuliani SpA, GlaxoSmithKline, Glenmark, Grunenthal, Immunocore, Immunometabolism, Istesso, Janssen, Lexicon, Lilly, Medarex, Merck, MSD, Napp Neovacs, NovoNordisk, Novartis, NPS Pharmaceuticals, Ocera, Otsuka, Pfizer, PharmOlam, Phillips, Proximagen, Qunitiles, Receptos, Robarts, Roche, Sandoz, Shire, Sigmoid Pharma, Takeda, Theravance, TiGenix, Topivert, UCB, Warner Chillcott, Vertex VHsquared, Vifor and Zeria; speaker for AbbVie, Amgen, Biogen, Ferring, Sandoz, Takeda, Zeria; and received research grants from AbbVie, Buhlmann, Lilly, MediAdd, UCB, Vifor and the Norman Collisson Foundation. PB has received educational grants from AbbVie, Janssens; speaker fees from AbbVie, Takeda and MSD; and advisory board fees from Abbvie, Hospira, Janssen, MSD, Mundipharma, Roche, Pfizer and Dr Falk Benelux. FB has received research grants from Abbvie, Chiesi, Ipsen, MSD, Roche, speakers and consultancy fees from Abbvie, Falk, Ferring, Janssen, Mundipharma, MSD, Pfizer, Takeda, and Vifor. TV has served as advisory member for Hospira, Pfizer and Takeda, has received lecture fees from Takeda. GN has served as 
a consultant/advisory board member/speaker for AbbVie, MSD, Takeda, Ferring, Vifor, Merck, Janssen and Pfizer. AA has served as a consultant or advisory member for AbbVie, Allergan, Amgen, Biogen, Celgene, Celltrion, Ferring, Hospira, Janssen, Lilly, MSD, Mundipharma, Pfizer, Samsung Bioepis, Sofar and Takeda, has received lecture fees from AbbVie, AstraZeneca, Chiesi, Ferring, Hospira, Medtronic, MSD, Mitsubishi Tanabe, Mundipharma, Nikkiso, Otsuka, Pfizer, Takeda, Tigenix and Zambon, has received research funding from MSD and Takeda. WR has served as a speaker for Abbott Laboratories, Abbvie, Aesca, Aptalis, Astellas, Centocor, Celltrion, Danone Austria, Elan, Falk Pharma GmbH, Ferring, Immundiagnostik, Mitsubishi Tanabe Pharma Corporation, MSD, Otsuka, PDL, Pharmacosmos, PLS Education, ScheringPlough, Shire, Takeda, Therakos, Vifor, Yakult; as a consultant for Abbott Laboratories, Abbvie, Aesca, Amgen, AM Pharma, Astellas, Astra Zeneca, Avaxia, Roland Berger $\mathrm{GmBH}$, Bioclinica, Biogen IDEC, Boehringer-Ingelheim, Bristol-Myers Squibb, Cellerix, Chemocentryx, Celgene, Centocor, Celltrion, Covance, Danone Austria, Elan, Ernest \& Young, Falk Pharma GmbH, Ferring, Galapagos, Genentech, Gilead, Grünenthal, ICON, Index Pharma, Inova, Janssen, Johnson \& Johnson, Kyowa Hakko Kirin Pharma, Lipid Therapeutics, Mallinckrodt, Medlmmune, Millenium, Mitsubishi Tanabe Pharma Corporation, MSD, Nestle, Novartis, Ocera, Otsuka, PDL, Pharmacosmos, Pfizer, Procter \& Gamble, Prometheus, Robarts Clinical Trial, Schering-Plough, Second Genome, Setpointmedical, Sigmoid, Takeda, Therakos, Tigenix, UCB, Vifor, Zyngenia and $4 \mathrm{SC}$; as an advisory board member for Abbott Laboratories, Abbvie, Aesca, Amgen, AM Pharma, Astellas, Astra Zeneca, Avaxia, Biogen IDEC, BoehringerIngelheim, Bristol-Myers Squibb, Cellerix, Chemocentryx, Celgene, Centocor, Celltrion, Danone Austria, Elan, Ferring, Galapagos, Genentech, Grünenthal, Inova, Janssen, Johnson \& Johnson, Kyowa Hakko Kirin Pharma, Lipid Therapeutics, Medlmmune, Millenium, Mitsubishi Tanabe Pharma Corporation, MSD, Nestle, Novartis, Ocera, Otsuka, PDL, Pharmacosmos, Pfizer, Procter \& Gamble, Prometheus, ScheringPlough, Second Genome, Setpointmedical, Takeda, Therakos, Tigenix, UCB, Zyngenia, and $4 \mathrm{SC}$; and has received research funding from Abbott Laboratories, Abbvie, Aesca, Centocor, Falk Pharma GmbH, Immundiagnsotik, and MSD. SJ and MB are employees of Medicus Economics LLC which was paid fees by AbbVie to conduct the research in the manuscript. EN, JP and W-JL are AbbVie employees and may own AbbVie stock and/or options. GRD'H is a consultant for AbbVie, ActoGeniX NV, AGI Therapeutics, Inc, Alba Therapeutics Corporation, Albireo, Alfa Wasserman, Amgen, AM-Pharma BV, Anaphore, Astellas, Athersys, Inc, Atlantic Healthcare Limited, Aptalis, BioBalance Corporation, Boehringer Ingelheim, Inc, Bristol-Myers Squibb, Celgene, Celek Pharmaceuticals, Cellerix SL, Cerimon Pharmaceuticals, ChemoCentryx, CoMentis, Cosmo Technologies, Coronado Biosciences, Cytokine PharmaSciences, Eagle Pharmaceuticals, Eisai Medical Research Inc, Elan Pharmaceuticals, enGene, Inc, Eli Lilly, EnteroMedics, Exagen Diagnostics, Inc, Ferring Pharmaceuticals, Flexion Therapeutics, Inc, Funxional Therapeutics Limited, Genzyme Corporation, Genentech, Gilead Sciences, Given Imaging, GlaxoSmithKline, Human Genome Sciences, Ironwood Pharmaceuticals, Janssen, KaloBios Pharmaceuticals, Inc, Lexicon Pharmaceuticals, Lycera Corporation, Meda Pharmaceuticals, Merck Research Laboratories, Merck Serono, Merck \& Co., Millennium, Nisshin Kyorin Pharmaceuticals Co, Ltd, Novo Nordisk A/S, NPS Pharmaceuticals, Optimer Pharmaceuticals, Orexigen Therapeutics, Inc, PDL BioPharma, Pfizer, Procter \& Gamble, Prometheus Laboratories, ProtAb Limited, PurGenesis Technologies, Inc, Receptos, Relypsa, Inc, Salient Pharmaceuticals, Salix Pharmaceuticals, Inc, Santarus, Shire Pharmaceuticals, Sigmoid Pharma Limited, Sirtris Pharmaceuticals, Inc (a GSK company), SLA Pharma (UK) Limited, Targacept, Teva Pharmaceuticals, Therakos, Tillotts Pharma AG, TxCell SA, UCB Pharma, Viamet Pharmaceuticals, Vascular Biogenics Limited (VBL) and Warner Chilcott UK Limited; has received speaker fees from AbbVie, Bristol-Myers Squibb and Janssen; and has received financial support for research from AbbVie, Bristol-Myers Squibb, Genentech, GlaxoSmithKline, Janssen, Millennium, Novartis, Pfizer, Procter \& Gamble Pharmaceuticals, Shire Pharmaceuticals and UCB Pharma.

\section{Patient consent for publication Not required.}

Provenance and peer review Not commissioned; externally peer reviewed.

Open access This is an open access article distributed in accordance with the Creative Commons Attribution Non Commercial (CC BY-NC 4.0) license, which permits others to distribute, remix, adapt, build upon this work non-commercially, and license their derivative works on different terms, provided the original work is properly cited, appropriate credit is given, any changes made indicated, and the use is non-commercial. See: http://creativecommons.org/licenses/by-nc/4.0/.

\section{ORCID iDs}

Peter Bossuyt http://orcid.org/0000-0003-4027-7365

Scott Johnson http://orcid.org/0000-0002-2648-1127

\section{REFERENCES}

1 Torres J, Mehandru S, Colombel J-F, et al. Crohn's disease. The Lancet 2017;389:1741-55

2 Kappelman MD, Rifas-Shiman SL, Porter CQ, et al. Direct health care costs of Crohn's disease and ulcerative colitis in US children and adults. Gastroenterology 2008;135:1907-13.
3 Peyrin-Biroulet L, Loftus EV, Colombel JF, et al. The natural history of adult Crohn's disease in population-based cohorts. Am J Gastroenterol 2010;105:289-97.

4 Lichtenstein GR, Yan S, Bala M, et al. Remission in patients with Crohn's disease is associated with improvement in employment and quality of life and a decrease in hospitalizations and surgeries. Am J Gastroentero/ 2004;99:91-6.

5 Gibson TB, Ng E, Ozminkowski RJ, et al. The direct and indirect cost burden of Crohn's disease and ulcerative colitis. J Occup Environ Med 2008;50:1261-72.

6 van der Valk ME, Mangen MJ, Leenders M, et al. Healthcare costs of inflammatory bowel disease have shifted from hospitalisation and surgery towards anti-TNF $\alpha$ therapy: results from the COIN study. Gut 2014;63:72-9.

7 Bassi A, Dodd S, Williamson P, et al. Cost of illness of inflammatory bowel disease in the UK: a single centre retrospective study. Gut 2004;53:1471-8.

8 Khanna R, Bressler B, Levesque BG, et al. Early combined immunosuppression for the management of Crohn's disease (REACT): a cluster randomised controlled trial. Lancet 2015;386:1825-34.

9 Lichtenstein GR, Feagan BG, Cohen RD, et al. Serious infections and mortality in association with therapies for Crohn's disease: TREAT registry. Clin Gastroenterol Hepatol 2006;4:621-30.

10 Osterman MT, Sandborn WJ, Colombel J-F, et al. Crohn's disease activity and concomitant immunosuppressants affect the risk of serious and opportunistic infections in patients treated with adalimumab. Am J Gastroenterol 1806;2016:111

11 Peyrin-Biroulet L, Sandborn W, Sands BE, et al. Selecting Therapeutic Targets in Inflammatory Bowel Disease (STRIDE): determining therapeutic goals for treat-totarget. Am J Gastroenterol 2015;110:1324-38.

12 Colombel JF, Panaccione $R$, Bossuyt $\mathrm{P}$, et al. Effect of tight control management on Crohn's disease (CALM): a multicentre, randomised, controlled phase 3 trial. Lancet 2018;390:2779-89.

13 Silverstein MD, Loftus EV, Sandborn WJ, et al. Clinical course and costs of care for Crohn's disease: Markov model analysis of a population-based cohort. Gastroenterology 1999;117:49-57.

14 Loftus EV, Johnson SJ, Yu AP, et al. Cost-effectiveness of adalimumab for the maintenance of remission in patients with Crohn's disease. Eur J Gastroenterol Hepatol 2009;21:1302-9.

15 National Instutute for Health and Care Excellence. Infliximab and adalimumab for the treatment of Crohn's disease [TA187]. 2010. https://www.nice.org.uk/Guidance/ TA187 (Accessed 19 Oct 2018).

16 Bodger K, Kikuchi T, Hughes D. Cost-effectiveness of biological therapy for Crohn's disease: Markov cohort analyses incorporating United Kingdom patient-level cost data. Aliment Pharmacol Ther 2009;30:265-74.

17 National Health Service. NHS Dictionary of Medicines and Devices $(\mathrm{dm}+\mathrm{d})$ database. http://dmd.medicines.org.uk (Accessed 10 Jan 2018).

18 National Instutute for Health and Care Excellence. Point-of-care and home faecal calprotectin tests for monitoring treatment response in inflammatory bowel disease. 2017. https://www.nice.org.uk/advice/mib132 (Accessed 19 Oct 2018).

19 Hunter R. Cost-effectiveness of point-of-care C-reactive protein tests for respiratory tract infection in primary care in England. Adv Ther 2015:32:69-85.

20 Hospital and Community Health Services. Unit Costs of Health and Social Care. Hospital and Community Health Services (HCHS) pay and price inflation index. 2017. http://www.pssru.ac.uk/pub/uc/uc2017/sources-of-information.pdf?label=uc2017sources-of-information (Accessed 19 Oct 2018).

21 Reilly MC, Gerlier L, Brabant Y, et al. Validity, reliability, and responsiveness of the work productivity and activity impairment questionnaire in Crohn's disease. Clin Ther 2008;30:393-404.

22 Office for National Statistics. Weekly average earnings from July 17, for the Whole economy. Average Weekly Earnings (nominal) - Total Pay (Great Britain, seasonally adjusted). 2018 https://www.ons.gov.uk (Accessed 19 Oct 2018).

23 Ware JE, Snow KK, Kosinski M, et al. SF-36 Health Survey Manual and Interpretation Guide. Boston, Mass: The Health Institude, New England Medical Center, 1993.

24 Brazier J, Roberts J, Deverill M. The estimation of a preference-based measure of health from the SF-36. J Health Econ 2002;21:271-92.

25 Brazier J, Roberts J, Tsuchiya A, et al. A comparison of the EQ-5D and SF-6D across seven patient groups. Health Econ 2004;13:873-84.

26 Buxton MJ, Lacey LA, Feagan BG, et al. Mapping from disease-specific measures to utility: an analysis of the relationships between the Inflammatory Bowel Disease Questionnaire and Crohn's Disease Activity Index in Crohn's disease and measures of utility. Value Health 2007:10:214-20.

27 Briggs A, Sculpher M, Claxton K. Decision modelling for health economic evaluation. Oxford, United Kingdom: Oxford University Press, 2006.

28 McCabe C, Claxton K, Culyer AJ. The NICE cost-effectiveness threshold. Pharmacoeconomics 2008;26:733-44.

29 Glick HA, Doshi JA, Sonnad SS, et al; Economic evaluation in clinical trials. Oxford, United Kingdom: Oxford University Press, 2014

30 Chandra A, Cutler D, Song Z. Who ordered that? The economics of treatment choices in medical care. Chapter in: Culyer AJ, Newhouse JP (eds), Handbook of health economics. Amsterdam, Netherlands: Elsevier, 2011.

31 Epstein AJ, Johnson SJ. Physician response to financial incentives when choosing drugs to treat breast cancer. Int J Health Care Finance Econ 2012;12:285-302. 